\title{
ANTICIPATORY GAZE AND ARGUMENT STRUCTURE BUILDING IN CHILDREN WITH SPECIFIC LANGUAGE IMPAIRMENT
}

\author{
MARIJAN PALMOVIĆa - INES GALIĆ JUŠIĆb \\ ${ }^{a}$ Laboratory for Psycholinguistic Research, University of Zagreb, Croatia \\ ${ }^{\mathrm{b}}$ Postgraduate Study Language and Cognitive Neuroscience, \\ University of Zagreb, Croatia \\ Corresponding author: Marijan Palmović, address: Gajdekova 36, \\ 10000, Zagreb, Croatia, e-mail: palmovic@erf.hr
}

\begin{abstract}
A listening paradigm was employed in an eye-tracking study to establish differences in argument structure building between children with typical language development (TLD) and children with Specific Language Impairment (SLI). The pictures presented to the participants triggered anticipatory eye movements due to the nature of their relation to the verb and its argument structure (syntactic or semantic). The difference between the two groups of children reveals the compensatory strategy of children with SLI.
\end{abstract}

Keywords: anticipatory gaze, Specific Language Impairment, argument structure, listening paradigm

\section{Introduction}

In recent years, eye tracking seems to have established itself as a valuable method in language processing research. It offers a number of dependent measures (like the moment of first fixation, gaze duration, number of regressive movements, etc.) and can be particularly useful in studying automatic processes that are involved in language comprehension. It can be used for studying any linguistic feature that has some cognitive relevance; i.e., any feature that can bring about a change in a behavioral measure.

Although eye tracking is relatively new in psycholinguistics, the study of eye movement and attention has been studied for more than a century. Basic principles discriminating between overt and covert atten- 
tion (Wright-Ward 2008) have been studied, defining the way attention directs the eye towards a stimulus. The "eye-mind hypothesis" (Carpenter-Just 1980) is of particular importance in language studies using eye tracking; the claim that the mind is processing what the eye is watching provides an opportunity to study temporal aspects of language processing.

In this study, a listening paradigm was used to study anticipatory gaze in two groups of participants, children with typical language development (TLD) and children with Specific Language Impairment (SLI). The listening paradigm usually consists of an auditorily presented sentence together with a set of pictures presented on the computer screen. The listening paradigm exploits the non-linguistic nature of visual stimuli in order to obtain information about the mapping between the sentence (syntactic information) and the referents of its parts (semantic information). It is based on the empirical finding that people generally look at the referents of the words they hear (Cooper 1974), or, more precisely, that the probability that the participant will look at a particular object rises if the object is mentioned (ibid.).

There is no general consensus on the interpretation of anticipatory gaze, i.e., a tendency that participants look ahead of what is mentioned in the sentence they hear in the headphones. It is generally recognized that it is related to verbs, not nouns; i.e., that participants tend to look at the arguments of the verbs at the moment they hear the verb. They do not wait until the noun is actually mentioned. This is why there is no inconsistency between the existence of anticipatory gaze and Cooper's principle referred to above or the "eye-mind" hypothesis. However, there is no agreement on what it is that triggers anticipatory gaze. According to Altmann and Kamide (Altmann-Kamide 1999), it is the building up of the syntactic structure (the argument structure of the verb). They confronted the participants with sentences involving two kinds of verb, e.g., move and eat; when the first verb was mentioned, the participants would turn their gaze to a knife on a kitchen table; when the verb eat was mentioned, they would look at the cake. On the other hand, Boland (2007) showed that expectations related to world knowledge are decisive; in sentences such as A girl is sleeping, participants turned their gaze to the picture of a bed, although bed is not part of the argument structure of the verb. Finally, anticipatory gaze was obtained in sentences such as Take the tall glass... with the picture presentation of a glass and e.g., a spoon, a fork and a bowl. A participant would look at the glass as soon 
as (s)he heard the word tall (Sedivy et al. 1999). Anticipatory gaze was not triggered by the verb here, but by the adjective; the only thing these words have in common is that they are, in a sense, non-referential, i.e., they do not refer to single objects, but to properties.

Specific Language Impairment has often been studied in the context of theoretical linguistics as psycholinguistic evidence for a linguistic theory. However, the mechanism that leads to impaired language is not known. Is it auditory perception deficit (Tallal 1976), working memory deficit (Gathercole 2006), or a deficit in some specific part of the language system like phonology (Leonard 1998), morphology (Rice et al. 1995), or syntax (Clahsen 1989)? There is no agreement about the specificity of SLI, either; while some claim that language is impaired due to a general slowing in the child's development (Kail 1994), others point to an impairment of the general learning mechanism that prevents the affected children from learning the critical mass of the words necessary for generalizing rules of the language (Conti-Ramsden--Jones 1997).

Although language-processing difficulties of children with SLI are in the focus of this paper, children with SLI will also help in a theoretical dispute about the nature of anticipatory gaze, making the aims of the study twofold. Since anticipatory gaze seems to be, first, a robust finding and, second, very sensitive to the particular stimuli and tasks given to the participants, having two groups of participants might be a fruitful approach to the problem. Namely, if the experiment manipulates syntactic and semantic processing in two groups of participants (children with TLD and SLI), it can be assumed that the switch between conditions will affect the two groups differently. Therefore, in this study two questions will be addressed: Is it syntactic or semantic information that guides the anticipatory gaze? And if it is the former, will children with an obvious deficit in grammatical knowledge show a different pattern or make use of semantic instead of syntactic information (compensatory strategy)? In other words, will children with SLI have just lower scores (smaller anticipatory gazes) or will they behave differently than children with TLD? It is this possible difference that will tell us about the nature of the information that is guiding their gaze in the process of resolving sentence meaning. 


\section{Materials and methods}

\subsection{Participants}

Fourteen children between 8 and 10 years were included in this study. Seven children had been previously diagnosed with SLI ( 4 boys and 3 girls). For this study, the diagnosis was confirmed in the Clinical Unit of our laboratory and was based on the difference in performance on language and non-language tests. All children were tested on the Wechsler non-verbal intelligence test, WISC (Wechsler 1974) and on the Croatian version of the Peabody Picture Vocabulary Test-III (Dunn et al. 2009). These are the only relevant standardized tests available for Croatian. Other materials used for the diagnosis are various non-standardized test materials that measure all aspects of language: phonology, morphology, and syntax. For example, morphology is tested on a set of questions that require an answer in a particular noun or verb form depending on a picture (e.g., a picture of a king is presented while the experimenter says "This is a king". When there is a picture of a queen without the king, the experimenter says "Here, there is no..." expecting a noun in the genitive; when several kings are presented in a picture, the sentence would be "Here are the..." expecting the noun in the nominative plural, etc.). The tests are tuned to younger, kindergarten-age subjects, so children with TLD aged 8 to 10 generally give correct answers to nearly all questions; only some paradigms with labile $a$ (nom. peta $k$ 'Friday', dat. petk-u) or long vs. short plural (i.e., with or without the infix -ov-, e.g., sing. slon 'elephant', pl. slon-ov-i vs. sing. konj 'horse', pl. konj-i) are sometimes still not acquired at that age. Unfortunately, only scarce data are available for children aged 8 to 10; the database that is continuously being updated now contains only 30 children of the appropriate age range. On average, they gave correct answers to 38 out of 40 questions with a standard deviation of 3.6. Therefore, a score below 34 correct answers would place a child into the "below 1 SD group". However, the children with SLI picked for this study differed up to $-3 \mathrm{SD}$; this result should be taken with caution because it is at least partly affected by the ceiling effect in children with TLD aged 8 to 10. All children with SLI had been in therapy for at least two years. 


\subsection{Materials}

Five sentences were used with ten slides in two experimental conditions that can be labeled as "syntactic" and "semantic", respectively. This means that the experimental conditions varied slides, not sentences. In other words, each sentence was presented twice, each time with a different slide as in Figure 1. Each slide contained three pictures.

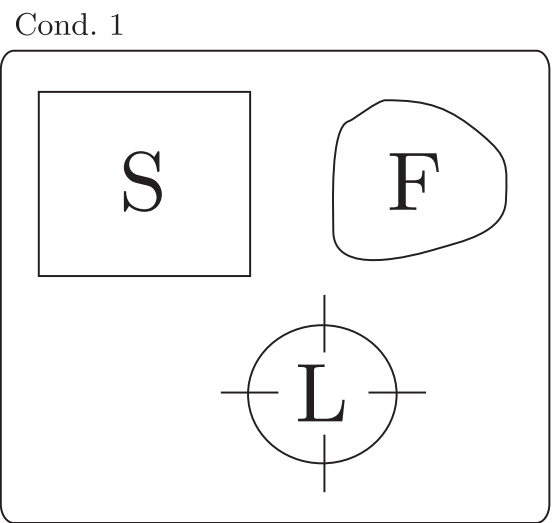

The $F$ is doing something to $L$.
Cond. 2

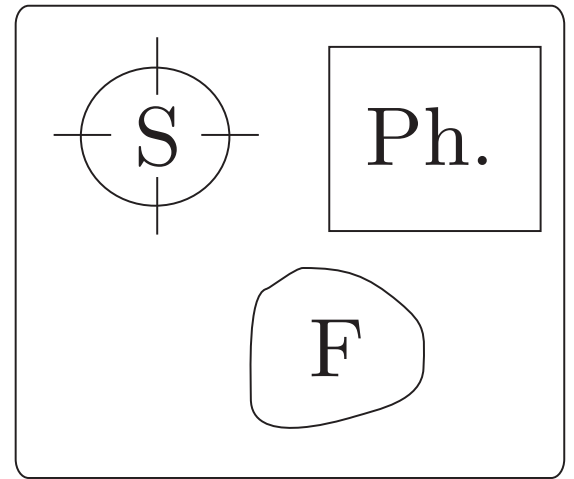

The $F$ is doing something to $L$.

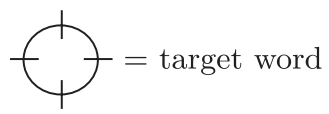

Fig. 1

A schematic view of the experimental conditions. $\mathrm{F}=$ picture related to the first word, $\mathrm{L}=$ picture related to the last word, $\mathrm{S}=$ picture related to a semantically similar word (not mentioned in the sentence), $\mathrm{Ph} .=$ picture related to a phonologically similar word to $\mathrm{F}$.

1. The "syntactic" condition. In the first condition the slides contained two pictures mentioned in the sentence: a picture representing the first noun and a picture representing the last word of the sentence (also a noun). The third picture represented a word that was semantically similar to the last word of the sentence. So, in the "syntactic" condition, each sentence was accompanied by pictures of the F-word (first word), L-word (last word) and the S-word (semantically related, not in gender agreement with the verb). The L-word was the target word for the anticipatory gaze measurement and agreed with the verb in gender. 
2. The "semantic" condition. The last word of the sentence (the L-word) was not presented in a picture in the "semantic" condition; instead, a picture representing a word that was phonologically similar to the first word of the sentence was presented (the $\mathrm{Ph}$-word). This picture was meant to represent an independent criterion for possible gaze orientation and simply to be a more probable choice for the gaze than a random picture. This way all the pictures on slides in both conditions had some reason for their appearance: syntactic, semantic or phonological. The two conditions - "syntactic" and "semantic" - are schematically given in Figure 1.

Only pictures represented by the letters $\mathrm{F}$ and L were actually mentioned in the corresponding sentence and $\mathrm{L}$ was always an argument of the verb (direct object or subject). The L-picture was missing in the second condition; instead, the target S-picture was added. The target word was the word for which anticipatory gaze was measured. For example, sentence (1) was presented auditorily together with a picture of a parking place (F-word), a steering wheel (S-word) and a tire (L-word) in the "syntactic" condition; the bottom line shows the onset of each word in the sentence.

(1) Pri parkiranju je automobilu stradala guma.

by parking is car.dat damage.part.fem tire.nom.fem

'While parking, a tire on a car has been damaged.'
69259
11481332
2138
$2842 \mathrm{~ms}$

The target word was the L-word guma 'tire'. In the second condition, the same sentence was given with the pictures of a park (phonologically similar), a parking place and a steering wheel (semantically similar target word). The Croatian word for steering wheel is volan, which is a masculine noun; therefore, it could not be expected after a verb in the feminine form, i.e., it could not be regarded as a grammatically plausible argument of the verb.

\subsection{Procedure}

Pictures were presented in a random order; i.e., each slide had a different order of the pictures. The beginning of each sentence was synchronized with the appearance of its slide. In addition, four filler sentences were presented with pictures presenting various words of the sentences (not 
necessarily the arguments of the verb). Each sentence lasted up to 3.5 seconds. The onset of the last word of the sentence was measured and taken into account in the calculation of anticipatory gaze. Each slide was presented for 5 seconds on the computer screen. A pause of 3 seconds was inserted between slides. The slides themselves were also presented in a random order. The whole experiment was programmed in E-prime (Schneider et al. 2002). The experiment lasted for about 10 minutes, from beginning (calibration) to completion.

The participants sat in a chair in front of the SMI iView HiSpeed eye-tracker. Chin and forehead were fixed in the device and their gaze was calibrated on 13 points. The participants were given the instruction to listen to the sentences and watch the pictures on the computer screen; no task to perform was required. The device uses the infrared lamp and infrared camera to measure the difference between the foveal reflection of the lamp and the center of the pupil. The device has a sampling rate of $500 \mathrm{~Hz}$ and precision of $0.25^{\circ}$ to $0.5^{\circ}$ of the visual angle. The fixations and saccades are detected automatically, based on an algorithm that detects saccades as "primary events", using speed as a parameter. Microsaccades are automatically grouped into fixations. Various dependent measures are available: fixation duration, first fixation latency, number of entries in a region, etc. Data that contained too many artifacts were excluded from the dataset; the exclusion was done to preserve the equal number of data points throughout the dataset (if, for example, a second sentence from Condition 1 had to be excluded, the same sentence was excluded from Condition 2). The main sources of the artifacts were blinks and head movements. Blinks were treated automatically; the software treats the blinks as "no data" periods. Small head movements are compensated for in the device; however, if a participant moves his/her head away from the device, calibration might be lost. This, in turn, results in data loss, i.e., in the exclusion of the data.

\section{Results}

In this experiment a very robust effect of anticipatory gaze was obtained in the group of children with typical language development (TLD). Anticipatory gaze was calculated by subtracting the moment of the first fixation on the target picture from the onset of the word for each sentence. The mean time of anticipatory gaze was about two and a half seconds; this means that the participants (at least, children with TLD) 
proceeded to the second argument even before they had heard the verb, i.e., right after they looked at the picture representing the first word of the sentence. This might be due to the high predictability of the target word (represented by the L-picture) that was the subject of the sentence (three times) or its direct object (twice). These results are in accordance with numerous studies in sentence processing showing that the relation between the nouns can trigger a neurocognitive effect (e.g., an ERP effect) before the verb itself is encountered (see Bornkessel SchlesewskySchlesewsky 2009) as, for example, in verb final languages. Anticipatory gaze was obtained in the group of children with SLI as well, although being only about a second and a half.

Two-way ANOVA was performed with two fixed factors (groupTLD/SLI and condition - "syntactic"/"semantic"). Two dependent variables were measured: anticipatory gaze and average fixation duration on the target picture. There was a significant main effect of group (TLD/ SLI) found on the anticipatory gaze variable, $F(1,56)=10.48, p<0.01$. The main effect of condition was non-significant on the same variable, $F(1,56)=0.25, p=0.617$. Finally, there was non-significant interaction between the group the children belonged to and the syntactic vs. semantic condition, $F(1,56)=1.19, p=0.219$. The results are shown in Figure 2 .

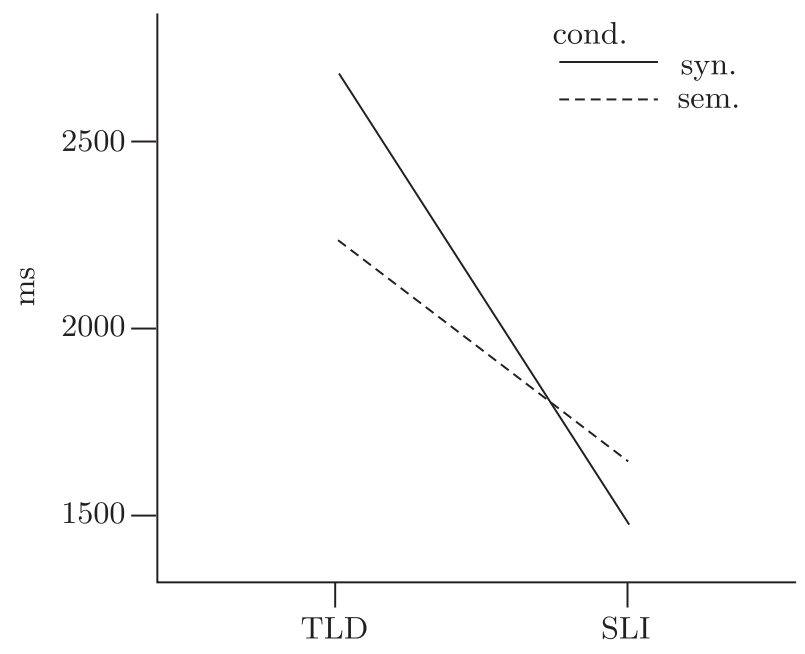

Fig. 2

Anticipatory gaze for TLD and SLI children in the syntactic and semantic conditions 
There was also a significant main effect of group for the average gaze duration on the target word, $F(1,56)=4.27, p<0.05$. There was a nonsignificant main effect of condition, $F(1,56)=1.44, p=0.24$. Finally, there was a non-significant effect of interaction of group and condition, $F(1,56)=0.314, p=0.58$. The results for average gaze duration are shown in Figure 3.

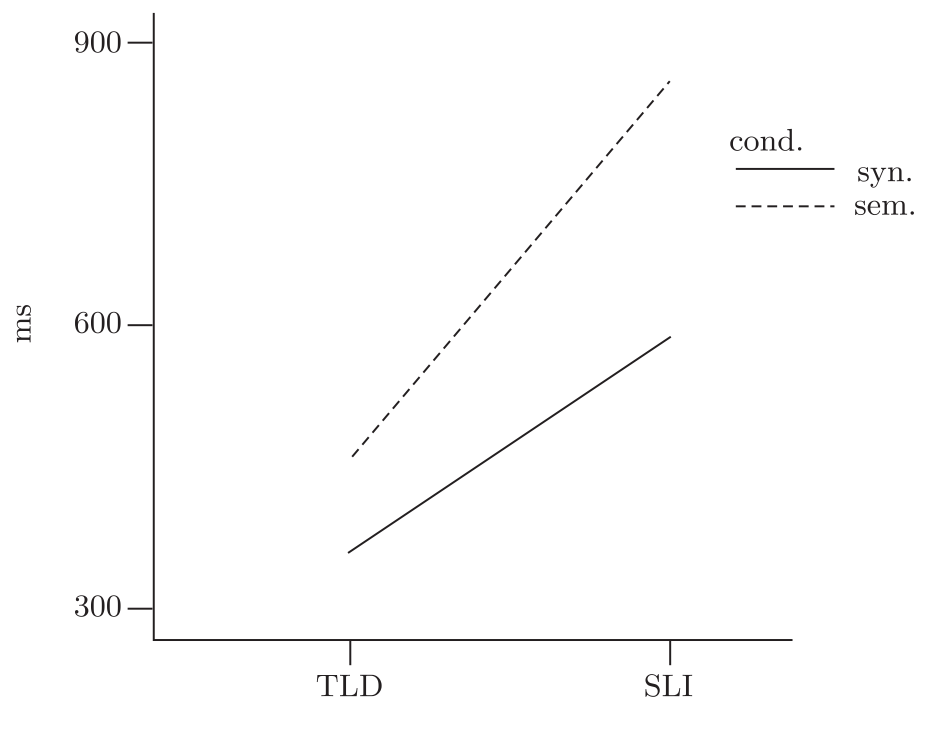

Fig. 3

Average duration of gaze on the target word for children with TLD and SLI in the syntactic and semantic condition

Both Figures 2 and 3 clearly show the main effect of group, i.e., the difference in language processing between the two groups of children. The difference tends to be greater for anticipatory gaze in the syntactic condition, i.e., when the argument of the verb is represented with a picture. The absence of the picture representing the last word of a sentence tends to affect children with TLD more than children with SLI. The mean values illustrate this point (Table 1).

However, as neither the difference between the conditions nor group $*$ condition interaction are significant, this can only be regarded as a trend.

The average duration of gaze on the target word is a variable not related to argument structure building. We can interpret it as the time required for the recognition of the best referent. This is the closest interpretation to the claim that fixation duration roughly corresponds to 
Table 1

Mean values of anticipatory gaze in the TLD and SLI groups

\begin{tabular}{lcc}
\hline & Syntactic cond. & Semantic cond. \\
\hline TLD & 2697.7 & 2250.5 \\
SLI & 1483.0 & 1648.2 \\
\hline
\end{tabular}

Table 2

Mean values of average duration of gaze on the target picture in the TLD and SLI groups

\begin{tabular}{lcc}
\hline & Syntactic cond. & Semantic cond. \\
\hline TLD & 361.4 & 458.8 \\
SLI & 1590.8 & 858.9 \\
\hline
\end{tabular}

word retrieval in reading (Ehrlich-Rayner 1981). Table 2 shows the mean values for average gaze duration.

In contrast to anticipatory gaze, the absence of the picture representing the last word tends to affect children with SLI more than children with TLD on this variable.

\section{Discussion}

The results clearly show the differences in language processing between the two groups of children. Results show overall slower performance of children with SLI, but they also suggest that all aspects of language are not equally affected. The results indicate that argument structure building is relatively weaker (in relation to semantic information processing) in the group of children with SLI: not only did the difference between the "syntactic" and "semantic" conditions tend to affect children in the SLI group less than the TLD group, but the SLI group showed slightly stronger anticipatory looks in the "semantic" condition. This may be taken as a sign of a higher degree of reliance on semantic information in the reconstruction of sentence meaning in the group of children with SLI. Children with TLD tend to rely more on syntactic information. Reliance on semantics might also be a compensatory mechanism of children with SLI no matter what syntactic information is available. 
Finally, the results are consistent with the claim made by Altmann and Kamide (1999) that anticipatory eye movements reflect argument structure building. However, this claim should be weakened: it does represent these processes if the relevant information is available. If not, some other (semantic, contextual) processes will take place. In a way, the results are consistent with immediacy models of language processing (Hagoort 2005). In these models it is claimed that any linguistic information is processed immediately as it becomes available. Syntactic information is the fastest and most efficient; it goes ahead of the speaker's current position in a sentence. If it is not available, semantic information will do the job, but not as fast. Finally, further studies into the role of anticipatory gaze in language processing should make use of the fine-grained distinctions of syntactic processing in the different linguistic theories. If anticipatory gaze goes ahead of the verb, this finding can be interpreted in terms of relational structures (subject, object) or the syntax-semantics interface (thematic roles and their processing based on case information, for example) and not necessarily in terms of argument structure building.

\section{References}

Altmann, Gerry T. M.-Yuki Kamide 1999. Incremental interpretation at verbs: Restricting the domain of subsequent reference. In: Cognition $73: 247-64$.

Boland, Julie E. 2007. Linking eye movements to sentence comprehension in reading and listening. In: Manuel Carreiras - Charles Clifton Jr. (eds): The on-line study of sentence comprehension, 51-76. Psychology Press, New York.

Bornkessel Schlesewsky, Ina-Matthias Schlesewsky 2009. Processing syntax and morphology: A neurocognitive perspective. Oxford University Press, Oxford.

Carpenter, Patricia-Marcel Just 1980. A theory of reading: from eye fixation to comprehension. In: Psychology Review 89:329-54.

Clahsen, Harald 1989. The grammatical characterization of developmental dysphasia. In: Language $27: 897-920$.

Conti-Ramsden, Gina-Melanie Jones 1997. Verb use in specific language impairment. In: Journal of Speech, Language, and Hearing Research 40:1298-313.

Cooper, Roger M. 1974. The control of eye fixation by the meaning of spoken language: A new methodology for the real-time investigation of speech perception, memory, and language processing. In: Cognitive Psychology $6: 61-83$.

Dunn, Lloyd M. - Doug Dunn - Melita Kovačević - Nevena Padovan - Gordana Hržica Jelena Kuvač Kraljević - Maja Mustapić - Gordana Dobravac-Marijan Palmović 2009. Slikovni test rječnika PPVT-III-HR [Peabody Picture Vocabulary Test III., Croation version]. Naklada Slap, Jastrebarsko. 
Ehrlich, Susan F.- Keith Rayner 1981. Contextual effects on word recognition and eye movements during reading. In: Journal of Verbal Learning and Verbal Behavior $20: 641-55$.

Gathercole, Susan E. 2006. Nonword repetition and word learning: The nature of the relationship. In: Applied Psycholinguistics $27: 513-43$.

Hagoort, Peter 2005. On Broca, brain, and binding: a new framework. In: Trends in Cognitive Sciences $9: 416-23$.

Kail, Robert V. 1994. A method of studying the generalized slowing hypothesis in children with specific language impairment. In: Journal of Speech and Hearing Research $37: 418-21$.

Leonard, Laurence B. 1998. Children with specific language impairment. MIT Press, Cambridge MA.

Rice, Mabel L.-Kenneth Wexler-Patricia L. Cleave 1995. Specific language impairment as a period of optional infinitive. In: Journal of Speech, Language, and Hearing Research 38:850-63.

Schneider, Walter-Eschman Amy-Anthony Zuccolotto 2002. E-Prime user's guide. Psychology Software Tools, Pittsburgh.

Sedivy, Julie C.-Michael K. Tanenhaus-Craig G. Chambers-Gregory N. Carlson 1999. Achieving incremental semantic interpretation through contextual representation. In: Cognition 71:109-47.

Tallal, Paula 1976. Rapid auditory processing in normal and disordered language development. In: Journal of Speech and Hearing Research 19:561-71.

Wechsler, David 1974. Wechsler intelligence scales for children. Psychological Corporation, New York.

Wright, Richard D. - Lawrence M. Ward 2008. Orienting of attention. Oxford University Press, Oxford. 